\title{
Antimicrobial Activity Evaluation of New 1,3,4-oxadiazole Derivatives
}

\author{
Leyla Yurttaş, ${ }^{1, *}$, Emre Fatih Bülbül2, Sinem Tekinkoca ${ }^{3}$, Şeref Demirayak² \\ 'Faculty of Pharmacy, Department of Pharmaceutical Chemistry, Anadolu University, 26470 Eskişehir, Turkey \\ ${ }^{2}$ School of Pharmacy, Department of Pharmaceutical Chemistry, Medipol University, 34810, İstanbul, Turkey \\ ${ }^{3}$ Faculty of Pharmacy, Department of Pharmaceutical Microbiology, Anadolu University, 26470 Eskişehir, Turkey
}

\begin{abstract}
In this study, we have synthesized seven novel 2-[(5-(4-chlorobenzyl)-1,3,4-oxadiazol-2-yl)thio]- $\mathrm{N}$-(6-substituted benzothiazol-2-yl)acetamide derivatives (4ag) starting from ethyl 4-chlorophenyl acetate. The antimicrobial acitivity of the compounds was screened against seven Gram positive and Gram negative bacteria and four fungi species; Escherichia coli ATCC 25922, Escherichia coli ATCC 35218, Enterococcus faecalis ATCC 51299, Enterococcus faecalis ATCC 29212, Staphylococcus aureus, Klebsiella pneumoniae, Pseudomonas aeruginosa, Candida albicans, Candida krusei, Candida glabrata, Candida parapsilosis. Minumum inhibitor concentration (MIC) was calculated and compared with standard drugs, chloramphenicol and ketoconazole. Regarding the results of MIC, all compounds exhibited potency either at the higher concentrations or at the same concentrations compared with positive controls.
\end{abstract}

Keywords: 1,3,4-oxadiazole, benzothiazole, antibacterial, antifungal

\section{INTRODUCTION}

Infectious diseases are one of the most deadly diseases in the world ${ }^{1}$. Recently, the number of bacterial and fungal infections has rised dangereously ${ }^{2}$. Antibiotics and antifungals are the most important drug groups used in the treatment of bacterial and fungal infections. With the discovery of antibiotics, these drugs have begun to be used as main drugs in the treatment of infections. But over time, bacteria have begun to develop resistance because of frequent use and misuse. An uncontrolled increase in resistance of pathogenic microorganisms has wasted health resources ${ }^{3-5}$. This resistance to antimicrobial agents has shown

*Corresponding author: Leyla Yurttaş

E-mail address: lyurttas@anadolu.edu.tr 
that there is an urgent need for new treatment strategies and new antimicrobial drug discovery studies.

Heterocyclic chemistry was discovered in the early 180os. Heterocyclic members have an important area in organic chemistry because they have broad range of pharmacological effects ${ }^{6}$. Among them, oxadiazole is one of the prominent aromatic ring containing oxygen and nitrogen atoms. Due to its electronic and charge-transport properties it can be easily connected to various functional groups 7 . In addition, oxadiazoles have been extensively studied over recent years due to their different biological activities. This five member heterocyclic ring plays an important role in medicinal chemistrywhich exists in new molecules as pharmacophore groups ${ }^{8,9}$. Different classes of oxadiazoles have broad range of pharmacological activities such as antimalarial, anticonvulsant, analgesic, antimicrobial, antimycobacterial, antitumor, vasodilator, cytotoxic, hypolipidemic, antiproliferative, antifungal ${ }^{10-17}$. Some of prescribed agents possessing oxadiazole ring are antimicrobial furamizole ${ }^{6}$, antiretroviral agent raltegravir and antihypertensive agent nesapidil ${ }^{18}$ (Figure 1).

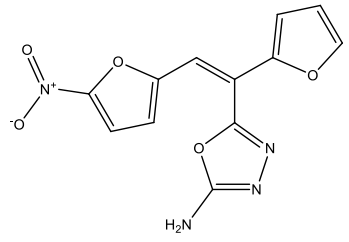

Furamizole

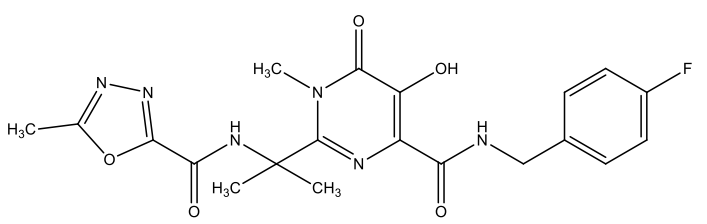

Raltegravir<smiles>COc1ccccc1N1CCN(CC(O)COc2cccc(-c3nnc(C)o3)c2)CC1</smiles>

Nesadipil

Figure 1. Some oxadiazole possessing drugs

Additionally, 1,3,4-oxadiazoles are good bioisosteres of amide and ester groups that exhibit different biological activities by making strong hydrogen bonds with different receptors ${ }^{10,11}$. On the other hand, 1,3,4-oxadizoles can react with the nucleophilic centers of microbial cells by reacting with the presence of the $-\mathrm{N}=$ C-O toxophoric group ${ }^{18}$.

There are several methods in the literature for the synthesis of 1,3,4-oxadiazoles. 
By using acid hydrazides, phosphorus oxychloride, sulfuric acid, and thionyl chloride, the oxadiazole ring was obtained in several steps ${ }^{14,15,19}$. However, the method of synthesis of 1,3,4-oxadiazoles by reaction of carboxylic acid and acid hydrazides is not a highly preferred method because it is expensive and requires a long time ${ }^{20}$.

In this study, we have synthesized seven novel compounds combining 5-(4-chlorobenzyl)-1,3,4-oxadiazol-2-thiol and 2-chloro- $N$-(2-benzothiazolyl) acetamide derivatives. The antimicrobial activity of the synthesized compounds was investigated against different microorganisms compared with standard drugs chloramphenicol and ketoconazole.

\section{METHODOLOGY}

\section{Chemistry}

All chemicals were purchased from Sigma-Aldrich Chemical Co (Sigma-Aldrich Corp., St. Louis, MO, USA) and Merck Chemicals (Merck KGaA, Darmstadt, Germany). All melting points (m.p.) were determined by MP9o digital melting point apparatus (Mettler Toledo, Ohio, USA) and were uncorrected. All reactions were monitored by thin-layer chromatography (TLC) using Silica Gel 60 F254 TLC plates (Merck KGaA, Darmstadt, Germany). Spectroscopic data were recorded with the following instruments: IR, Shimadzu Affinity $1 \mathrm{~S}$ spectrophotometer (Shimadzu, Tokyo, Japan); NMR, Agilent 300 MHz NMR spectrometer (Agilent technologies, California, USA), in DMSO- $d_{6}$, using TMS as internal standard; M+1 peaks were determined by Shimadzu 8040 LC/MS/MS system (Shimadzu, Tokyo, Japan). Elemental analyses were performed on a Leco 932 CHNS analyzer (Leco, Michigan, USA).

\section{Synthesis of ethyl 4-chlorophenyl acetate (1)}

4-Chlorophenyl acetic acid ( $0.40 \mathrm{~mol})$ was refluxed with excess ethanol for $12 \mathrm{~h}$ catalyzed with $\mathrm{H}_{2} \mathrm{SO}_{4}$. After TLC check, the solvent was evaporated under reduced pressure. The residue was dissolved in ethyl acetate, washed with salty water and dried with sodium sulfate. Ethyl acetate was evaporated under reduced pressure to gain ester compound (1).

\section{Synthesis of 2-(4-chlorophenyl)acetohydrazide (2)}

Ethyl 4-chlorophenyl acetate (0.25 mol) was dissolved in ethanol (150 ml). Hydrazine hydrate $(0.50 \mathrm{~mol})$ added and the mixture stirred in room temperature for $2 \mathrm{~h}$. After completion of reaction, the solvent was separated by filtration to acquire hydrazide compound $\mathbf{2}$. 


\section{Synthesis of 5-(4-chlorobenzyl)-1,3,4-oxadiazole-2-thiol (3)}

2-(4-Chlorophenyl)acetohydrazide (0.20 mol) was dissolved in ethanol (250 $\mathrm{ml})$. 0. $24 \mathrm{~mol}$ of potassium hydroxide was dissolved in ethanol (100 mL)and added to the mixture. Secondly, carbon disulfide ( $0.60 \mathrm{~mol})$ was added to the mixture, and it was refluxed for 5 hours. After this period, cold water and dilute $\mathrm{HCl}$ were added to the reaction mixture to gain product 3 .

Synthesis of 2-[(5-(4-chlorobenzyl)-1,3,4-oxadiazol-2-yl)thio]-N-(6substituted benzothiazol-2-yl)acetamidederivatives (4a-g)

5-(4-Chlorobenzyl)-1,3,4-oxadiazole-2-thiol (10 mmol) was dissolved in acetone $(50 \mathrm{~mL})$, potassium carbonate $(12 \mathrm{mmol})$ and appropriate 2-chloro- $\mathrm{N}$-(2-benzothiazolyl)acetamide derivatives were added to this solution and stirred for $12 \mathrm{~h}$ in room temperature. After TLC screening, the solvent was evaporated under reduced pressure then water was added to wash the resulting solid and the mixture was filtered, dried and recrystallized from ethanol to give final compounds 4a-g.

2-[(5-(4-Chlorobenzyl)-1,3,4-oxadiazol-2-yl)thio]-N-(benzothiazol2-yl)acetamide (4a)

Yield: $69 \%$. M.p. $231^{\circ} \mathrm{C}$.

${ }^{1} \mathrm{H}-\mathrm{NMR}\left(300 \mathrm{MHz}, \mathrm{DMSO}-\mathrm{d}_{6}, \mathrm{ppm}\right) 8: 4.27\left(2 \mathrm{H}, \mathrm{s},-\mathrm{CH}_{2}\right), 4.40\left(2 \mathrm{H}, \mathrm{s},-\mathrm{COCH}_{2}\right)$, 7.30-7.37 (5H, m, Ar-H), 7.46 (1H, t, $J=7.44 \mathrm{~Hz}, \mathrm{Ar}-\mathrm{H}), 7.78(1 \mathrm{H}, \mathrm{d}, J=7.95 \mathrm{~Hz}$, aromatic-H), $7.99(1 \mathrm{H}, \mathrm{d}, J=7.41 \mathrm{~Hz}$, aromatic-H), $12.74(1 \mathrm{H}, \mathrm{s},-\mathrm{NH})$.

${ }^{13} \mathrm{C}-\mathrm{NMR}$ (75 MHz, DMSO-d 6 , ppm) 8: $30.46\left(\mathrm{CH}_{2}\right), 36.01\left(\mathrm{CH}_{2}\right), 121.19(\mathrm{CH})$, $122.26(\mathrm{CH}), 124.23(\mathrm{CH}), 126.70(\mathrm{CH}), 129.10(\mathrm{CH}), 131.93(\mathrm{CH}), 132.48(\mathrm{C})$, 133.59 (C), 148.98 (C), 158.06 (C), 163.43 (C), 166.75 (C), 166.97 (C).

For $\mathrm{C}_{18} \mathrm{H}_{13} \mathrm{ClN}_{4} \mathrm{O}_{2} \mathrm{~S}_{2}$ calculated: $51.86 \% \mathrm{C}, 3.14 \% \mathrm{H}, 13.44 \% \mathrm{~N}$, found: $51.82 \%$ C, $3.15 \% \mathrm{H}, 13.47 \% \mathrm{~N}$.

HRMS (m/z): $[\mathrm{M}+\mathrm{H}]^{+}$calcd for $\mathrm{C}_{18} \mathrm{H}_{13} \mathrm{ClN}_{4} \mathrm{O}_{2} \mathrm{~S}_{2}: 417.0241$; found 417.0232.

\section{2-[(5-(4-Chlorobenzyl)-1,3,4-oxadiazol-2-yl)thio]-N-(6-methylben- zothiazol-2-yl)acetamide(4b)}

Yield: 72 \%. M.p. $244^{\circ} \mathrm{C}$.

${ }^{1} \mathrm{H}-\mathrm{NMR}$ (300 MHz, DMSO-d $\left.\mathrm{d}_{6}, \mathrm{ppm}\right) \mathrm{\delta}: 2.41\left({ }_{3} \mathrm{H}, \mathrm{s},-\mathrm{CH}_{3}\right), 4.27\left(2 \mathrm{H}, \mathrm{s},-\mathrm{CH}_{2}\right)$, $4.39\left(2 \mathrm{H}, \mathrm{s},-\mathrm{COCH}_{2}\right), 7.25-7.38(5 \mathrm{H}, \mathrm{m}, \mathrm{Ar}-\mathrm{H}), 7.66(1 \mathrm{H}, \mathrm{d}, J=8.22 \mathrm{~Hz}, \mathrm{Ar}-\mathrm{H})$, $7.78(1 \mathrm{H}, \mathrm{s}$, aromatic-H), $12.67(1 \mathrm{H}, \mathrm{s},-\mathrm{NH})$.

${ }^{13} \mathrm{C}-\mathrm{NMR}\left(75 \mathrm{MHz}, \mathrm{DMSO}-\mathrm{d}_{6}, \mathrm{ppm}\right)$ 8: $21.46\left(\mathrm{CH}_{3}\right), 30.46\left(\mathrm{CH}_{2}\right), 35.99\left(\mathrm{CH}_{2}\right)$, $121.83(\mathrm{CH}), 122.41(\mathrm{CH}), 123.35(\mathrm{CH}), 128.62(\mathrm{CH}), 129.10(\mathrm{CH}), 131.26(\mathrm{C})$, 
132.06 (C), 132.48 (C), 133.72 (C), 146.91 (C), 157.19 (C), 163.44 (C), 166.59 (C), 166.96 (C).

For $\mathrm{C}_{19} \mathrm{H}_{15} \mathrm{ClN}_{4} \mathrm{O}_{2} \mathrm{~S}_{2}$ calculated: $52.96 \% \mathrm{C}, 3.51 \% \mathrm{H}, 13.00 \% \mathrm{~N}$, found: $52.91 \%$ $\mathrm{C}, 3.52 \% \mathrm{H}, 13.04 \% \mathrm{~N}$.

HRMS (m/z): $[\mathrm{M}+\mathrm{H}]^{+}$calcd for $\mathrm{C}_{19} \mathrm{H}_{15} \mathrm{ClN}_{4} \mathrm{O}_{2} \mathrm{~S}_{2}: 431.0394$; found 431.0394 .

\section{2-[(5-(4-Chlorobenzyl)-1,3,4-oxadiazol-2-yl)thio]-N-(6-methoxy- benzothiazol-2-yl)acetamide (4c)}

Yield: $75 \%$. M.p. $241^{\circ} \mathrm{C}$.

${ }^{1} \mathrm{H}-\mathrm{NMR}\left(300 \mathrm{MHz}, \mathrm{DMSO}-\mathrm{d}_{6}, \mathrm{ppm}\right) 8: 3.80\left(3 \mathrm{H}, \mathrm{s},-\mathrm{OCH}_{3}\right), 4.26\left(2 \mathrm{H}, \mathrm{s},-\mathrm{CH}_{2}\right)$, $4.38\left(2 \mathrm{H}, \mathrm{s},-\mathrm{COCH}_{2}\right), 7.04(1 \mathrm{H}, \mathrm{dd}, J=8.42 \mathrm{~Hz}, J=2.58 \mathrm{~Hz}, \mathrm{Ar}-\mathrm{H}), 7.29-7.37$ (4H, m, Ar-H), $7.57(1 \mathrm{H}, \mathrm{s}$, aromatic-H), 7. $65(1 \mathrm{H}, \mathrm{d}, J=8.85 \mathrm{~Hz}, 12.61(1 \mathrm{H}, \mathrm{s}$, $-\mathrm{NH})$.

${ }^{13} \mathrm{C}-\mathrm{NMR}\left(75 \mathrm{MHz}, \mathrm{DMSO}-\mathrm{d}_{6}\right.$, ppm) $\delta: 30.46\left(\mathrm{CH}_{2}\right), 35.96\left(\mathrm{CH}_{2}\right), 56.10\left(\mathrm{OCH}_{3}\right)$, $121.79(\mathrm{CH}), 124.45(\mathrm{CH}), 128.62(\mathrm{CH}), 129.09(\mathrm{CH}), 131.26(\mathrm{CH}), 132.48(\mathrm{C})$, 133.25 (C), 133.59 (C), 143.00 (C), 156.00 (C), 156.74 (C), 163.45 (C), 166.44 (C), 166.95 (C).

For $\mathrm{C}_{19} \mathrm{H}_{15} \mathrm{ClN}_{4} \mathrm{O}_{3} \mathrm{~S}_{2}$ calculated: $51.06 \% \mathrm{C}, 3.38 \% \mathrm{H}, 12.54 \% \mathrm{~N}$, found: $51.11 \%$ $\mathrm{C}, 3.39 \% \mathrm{H}, 12.58 \% \mathrm{~N}$.

HRMS (m/z): $[\mathrm{M}+\mathrm{H}]^{+}$calcd for $\mathrm{C}_{19} \mathrm{H}_{15} \mathrm{ClN}_{4} \mathrm{O}_{3} \mathrm{~S}_{2}$ : 447.0347; found 447.0328.

2-[(5-(4-Chlorobenzyl)-1,3,4-oxadiazol-2-yl)thio]-N-(6-ethoxybenzothiazol-2-yl)acetamide (4d)

Yield: $68 \%$. M.p. $233^{\circ} \mathrm{C}$.

${ }^{1} \mathrm{H}-\mathrm{NMR}\left(300 \mathrm{MHz}, \mathrm{DMSO}-\mathrm{d}_{6}, \mathrm{ppm}\right) 8: 1.35\left(3 \mathrm{H}, \mathrm{t}, J=6.93 \mathrm{~Hz},-\mathrm{CH}_{3}\right), 4.07(2 \mathrm{H}$, $\left.\mathrm{q}, J=6.90 \mathrm{~Hz},-\mathrm{OCH}_{2}\right), 4.27\left(2 \mathrm{H}, \mathrm{s},-\mathrm{CH}_{2}\right), 4.37\left(2 \mathrm{H}, \mathrm{s},-\mathrm{COCH}_{2}\right), 7.03(1 \mathrm{H}, \mathrm{dd}, J=$ $8.70 \mathrm{~Hz}, J=2.49 \mathrm{~Hz}, \mathrm{Ar}-\mathrm{H}), 7.30-7.37(4 \mathrm{H}, \mathrm{m}, \mathrm{Ar}-\mathrm{H}), 7.56$ (1H, s, aromatic-H), 7. $65(1 \mathrm{H}, \mathrm{d}, J=8.82 \mathrm{~Hz}, 12.59(1 \mathrm{H}, \mathrm{s},-\mathrm{NH})$.

${ }^{13} \mathrm{C}-\mathrm{NMR}\left(75 \mathrm{MHz}\right.$, DMSO-d 6 , ppm) 8: $15.15\left(\mathrm{CH}_{3}\right), 30.46\left(\mathrm{CH}_{2}\right), 35.95\left(\mathrm{CH}_{2}\right)$, 64.08 $\left(\mathrm{OCH}_{2}\right), 121.80(\mathrm{CH}), 124.80(\mathrm{CH}), 129.10(\mathrm{CH}), 131.26(\mathrm{CH}), 132.47(\mathrm{C})$, 133.25 (C), 133.60 (C), 135.12 (C), 142.96 (C), 155.96 (C), 163.45 (C), 166.39 (C), 166.96 (C).

For $\mathrm{C}_{20} \mathrm{H}_{17} \mathrm{ClN}_{4} \mathrm{O}_{3} \mathrm{~S}_{2}$ calculated: $52.11 \% \mathrm{C}, 3.72 \% \mathrm{H}, 12.15 \% \mathrm{~N}$, found: $52.15 \% \mathrm{C}$, $3.73 \% \mathrm{H}, 12.18 \% \mathrm{~N}$.

HRMS (m/z): $[\mathrm{M}+\mathrm{H}]^{+}$calcd for $\mathrm{C}_{20} \mathrm{H}_{17} \mathrm{ClN}_{4} \mathrm{O}_{3} \mathrm{~S}_{2}: 461.0503$; found 461.0494 . 
2-[(5-(4-Chlorobenzyl)-1,3,4-oxadiazol-2-yl)thio]-N-(6-chlorobenzothiazol-2-yl)acetamide (4e)

Yield: 71 \%. M.p. $247^{\circ} \mathrm{C}$.

${ }^{1} \mathrm{H}-\mathrm{NMR}$ (3OO MHz, DMSO-d 6 , ppm) $\delta: 4.28$ (2H, s, $\mathrm{CH}_{2}$ ), $4.41\left(2 \mathrm{H}, \mathrm{s},-\mathrm{CH}_{2} \mathrm{CO}\right.$ ), 7.31-7.37 (4H, m, Ar-H), 7.47 ( $1 \mathrm{H}, \mathrm{dd}, J=8.70 \mathrm{~Hz}, J=2.22 \mathrm{~Hz}, \mathrm{Ar}-\mathrm{H}), 7.77(1 \mathrm{H}$, d, $J=8.64 \mathrm{~Hz}$, aromatic-H), $8.14(1 \mathrm{H}, \mathrm{d}, J=2.13 \mathrm{~Hz}, \mathrm{Ar}-\mathrm{H}), 12.85(1 \mathrm{H}, \mathrm{s},-\mathrm{NH})$.

${ }^{13} \mathrm{C}-\mathrm{NMR}\left(75 \mathrm{MHz}, \mathrm{DMSO}-\mathrm{d}_{6}, \mathrm{ppm}\right) \delta: 30.46\left(\mathrm{CH}_{2}\right), 35.97\left(\mathrm{CH}_{2}\right), 121.98(\mathrm{CH})$, 122.39 (CH), $127.05(\mathrm{CH}), 128.29(\mathrm{C}), 128.63(\mathrm{C}), 129.08(\mathrm{CH}), 131.24(\mathrm{CH})$, 132.48 (C), 133.57 (C), 147.84 (C), 158.95 (C), 163.40 (C), 166.97 (C).

For $\mathrm{C}_{18} \mathrm{H}_{12} \mathrm{Cl}_{2} \mathrm{~N}_{4} \mathrm{O}_{2} \mathrm{~S}_{2}$ calculated: $47.90 \% \mathrm{C}, 2.68 \% \mathrm{H}, 12.41 \% \mathrm{~N}$, found: $47.81 \%$ $\mathrm{C}, 2.69 \% \mathrm{H}, 12.45 \% \mathrm{~N}$.

$\operatorname{HRMS}(\mathrm{m} / \mathrm{z})$ : $[\mathrm{M}+\mathrm{H}]^{+}$calcd for $\mathrm{C}_{18} \mathrm{H}_{12} \mathrm{Cl}_{2} \mathrm{~N}_{4} \mathrm{O}_{2} \mathrm{~S}_{2}: 450.9851$; found 450.9832 .

2-[(5-(4-Chlorobenzyl)-1,3,4-oxadiazol-2-yl)thio]-N-(6-florobenzothiazol-2-yl)acetamide (4f)

Yield: 69 \%. M.p. $241^{\circ} \mathrm{C}$.

${ }^{1} \mathrm{H}-\mathrm{NMR}$ (300 MHz, DMSO-d 6 , ppm) $\delta: 4.26$ (2H, s, $\mathrm{CH}_{2}$ ), 4.39 (2H, s, $-\mathrm{CH}_{2} \mathrm{CO}$ ), 7.27-7.36 (5H, m, Ar-H), 7.76-7.81 (1H, m, Ar-H), 7.91(1H, dd, $J=8.70 \mathrm{~Hz}, J=$ $2.64 \mathrm{~Hz}$, aromatic-H), $12.76(1 \mathrm{H}, \mathrm{s},-\mathrm{NH})$.

${ }^{13} \mathrm{C}-\mathrm{NMR}\left(75 \mathrm{MHz}, \mathrm{DMSO}-\mathrm{d}_{6}, \mathrm{ppm}\right)$ 8: $30.46\left(\mathrm{CH}_{2}\right), 35.94\left(\mathrm{CH}_{2}\right), 108.55(\mathrm{C})$, $108.90(\mathrm{C}), 114.68(\mathrm{C}), 115.00(\mathrm{CH}), 122.27(\mathrm{CH}), 122.40(\mathrm{CH}), 129.09(\mathrm{CH})$, $131.26(\mathrm{CH}), 132.47$ (C), 133.59 (C), 157.62 (C), 160.80 (C), 163.41 (C), 166.85 (C), 166.97 (C).

For $\mathrm{C}_{18} \mathrm{H}_{12} \mathrm{ClFN}_{4} \mathrm{O}_{2} \mathrm{~S}_{2}$ calculated: $49.71 \% \mathrm{C}, 2.78 \% \mathrm{H}, 12.88 \% \mathrm{~N}$, found: $49.81 \%$ $\mathrm{C}, 2.77 \% \mathrm{H}, 12.84 \% \mathrm{~N}$.

$\operatorname{HRMS}(\mathrm{m} / \mathrm{z}):[\mathrm{M}+\mathrm{H}]^{+}$calcd for $\mathrm{C}_{18} \mathrm{H}_{12} \mathrm{ClFN}_{4} \mathrm{O}_{2} \mathrm{~S}_{2}: 435.0147$; found 435.0137.

2-[(5-(4-Chlorobenzyl)-1,3,4-oxadiazol-2-yl)thio]-N-(6-nitrobenzothiazol-2-yl)acetamide (4g)

Yield: 73 \%. M.p. $245^{\circ} \mathrm{C}$.

${ }^{1} \mathrm{H}-\mathrm{NMR}\left(3 \mathrm{OO} \mathrm{MHz}, \mathrm{DMSO}-\mathrm{d}_{6}, \mathrm{ppm}\right) \delta: 4.26\left(2 \mathrm{H}, \mathrm{s}, \mathrm{CH}_{2}\right), 4.43\left(2 \mathrm{H}, \mathrm{s},-\mathrm{CH}_{2} \mathrm{CO}\right)$, 7.29-7.37 (4H, m, Ar-H), 7.92 (1H, d, $J=8.97 \mathrm{~Hz}, \mathrm{Ar}-\mathrm{H}), 8.28(1 \mathrm{H}, \mathrm{dd}, J=8.70$ $\mathrm{Hz}, J=2.43 \mathrm{~Hz}$, aromatic-H), $13.15(1 \mathrm{H}, \mathrm{s},-\mathrm{NH})$.

${ }^{13} \mathrm{C}-\mathrm{NMR}\left(75 \mathrm{MHz}, \mathrm{DMSO}-\mathrm{d}_{6}, \mathrm{ppm}\right) \delta: 30.46\left(\mathrm{CH}_{2}\right), 36.03\left(\mathrm{CH}_{2}\right), 119.61(\mathrm{CH})$, $121.30(\mathrm{CH}), 122.30(\mathrm{CH}), 129.08(\mathrm{CH}), 131.24(\mathrm{CH}), 132.47(\mathrm{C}), 132.68(\mathrm{C})$, 133.57 (C), 143.60 (C), 153.83 (C), 163.35 (C), 163.60 (C), 167.00 (C), 167.56 (C). 
For $\mathrm{C}_{18} \mathrm{H}_{12} \mathrm{ClN}_{4} \mathrm{O}_{4} \mathrm{~S}_{2}$ calculated: $46.81 \% \mathrm{C}, 2.62 \% \mathrm{H}, 15.16 \% \mathrm{~N}$, found: $46.89 \%$ C, $2.63 \% \mathrm{H}, 15.21 \% \mathrm{~N}$.

HRMS (m/z): [M+H] $]^{+}$calcd for $\mathrm{C}_{18} \mathrm{H}_{12} \mathrm{ClN}_{4} \mathrm{O}_{4} \mathrm{~S}_{2}$ : 462.0092; found 462.0084.

\section{Antimicrobial activity}

Antimicrobial activity against Escherichia coli (ATCC 25922), Escherichia coli (ATCC 35218), Enterococcus faecalis (ATCC 51299), Enterococcus faecalis (ATCC 29212), Staphylococcus aureus (ATCC 22019), Klebsiella pneumoniae (ATCC 700603), Pseudomonas aeruginosa (ATCC 27853), Candida albicans (ATCC 24433), Candida krusei (ATCC 6258), Candida glabrata (ATCC 90030), Candida parapsilosis (ATCC 90030) was determined by the microbroth dilutions technique using the Clinical Laboratory Standarts Institute (CLSI) recommendations ${ }^{21}$.

The lowest concentration that completely inhibited growth of the microorganism was defined as the minimum inhibitor concentration (MIC). MIC was screened and the results were compared to chloramphenicol and ketoconazole as positive controls. Each experiment was replicated twice.

\section{RESULTS AND DISCUSSION}

\section{Chemistry}

In this study, we have synthesized 2-[(5-(4-chlorobenzyl)-1,3,4-oxadiazol-2-yl) thio]- $N$-(6-substituted benzothiazol-2-yl)acetamide derivatives (4a-g) with four step synthetic procedure as shown Scheme 1. In the first step, compound 1 was synthesized by reacting 4-chlorophenyl acetic acid with $\mathrm{H}_{2} \mathrm{SO}_{4}$ in ethanol at reflux conditions. In the second step, compound $\mathbf{2}$ was synthesized by reacting 4-chlorophenyl acetate with hydrazine hydrate in ethanol at the room temperature. In the third step, compound $\mathbf{3}$ was synthesized by reacting 2-(4-chlorophenyl)acetohydrazide with potassium hydroxide and carbon disulfide in ethanol at the reflux conditions. In the last step, compounds $\mathbf{4 a - g}$ were synthesized by reacting 5-(4-chlorobenzyl)-1,3,4-oxadiazol-2-thiol with 2-chloro- $N$-(2-benzothiazolyl)acetamide derivatives in acetone at room temperature. The gained raw products were crystallized from ethanol.

In ${ }^{1} \mathrm{H}-\mathrm{NMR}$ spectra of the synthesized compounds (4a-g), peaks of acetamide (- $\mathrm{CH}_{2} \mathrm{CONH}-$ ) moiety belongs to methylene and amide protons were identified at about 4.37-4.43 ppm and 12.59-13.15 ppm, respectively. If we look at the characteristic ${ }^{1} \mathrm{H}-\mathrm{NMR}$ properties of the molecule, we detected peaks of methyl group $\left(-\mathrm{CH}_{3}\right)$ at $2.41 \mathrm{ppm}$ for $\mathbf{4 b}$, peaks of methoxy group $\left(-\mathrm{OCH}_{3}\right)$ at $3.80 \mathrm{ppm}$ for $4 \mathbf{c}$. For compound $\mathbf{4 d}$, peaks of methyl $\left(-\mathrm{CH}_{3}\right)$ and methylene group $\left(-\mathrm{OCH}_{2}\right)$ were 
seen at $1.35 \mathrm{ppm}$ and $4.07 \mathrm{ppm}$ as broad singlet peaks. In the ${ }^{13} \mathrm{C}-\mathrm{NMR}$ spectra, all carbons were determined between $15.15-167.56 \mathrm{ppm}$ range. The peaks resonated at about $30.46 \mathrm{ppm}$ and 35.94-36.03 ppm were assigned to $-\mathrm{SCH}_{2}-$ and $-\mathrm{CCH}_{2}$ - carbons, respectively. In the ${ }^{13} \mathrm{C}-\mathrm{NMR}$ spectra of the compounds $\mathbf{4 b}, \mathbf{4 c}$ and 4d, signals at 21.46, 56.10 and 15.15, 64.08 ppm were assigned to the carbon atoms of methyl, methoxy and ethoxy groups on benzothiazole ring. In aromatic region, signals with higher values were determined for the carbon atoms of the heterocyclic rings. In the MS spectra of the compounds, $\mathrm{M}^{+}$peaks were observed in agreement with molecular weights of the compounds. Elemental analysis for $\mathrm{C}, \mathrm{H}, \mathrm{O}$ atoms were within $+/-0.4 \%$ of the theoretical values.
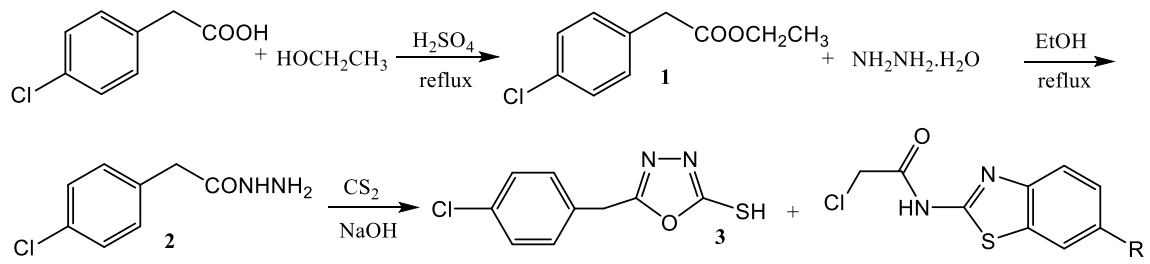

R: $\mathrm{H}$

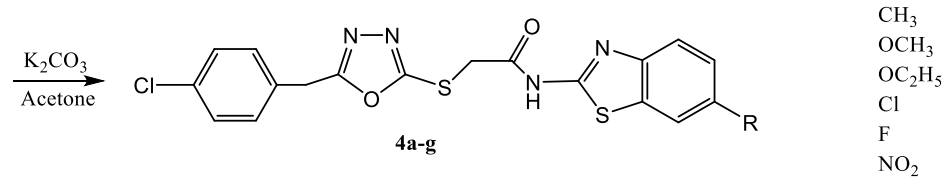

Scheme 1. The synthesis of the compounds (4a-g).

\section{Biology}

All synthesized compounds were tested for determining their antimicrobial activity against seven Gram positive and Gram negative bacterial and four fungal microorganisms; E.coli ATCC 25922, E. coli ATCC 35218, E. faecalis ATCC 51299, E. faecalis ATCC 29212, S. aureus, K.pneumoniae, P. aeruginosa, $C$. albicans, C.krusei, C.glabrata, C. parapsilosis. MIC values were determined against standard drugs, ketoconazole and chloramphenicol and represented in Table 1. MIC values of the compounds were found between $50-100 \mu \mathrm{g} / \mathrm{ml}$ and they were identified between $12.5-50 \mu \mathrm{g} / \mathrm{ml}$ for reference drugs. Compound $4 \mathbf{a}$ showed antimicrobial activity against all microorganisms at $50 \mu \mathrm{g} / \mathrm{ml}$ concentration. Compounds $\mathbf{4 b}, \mathbf{4 c}, \mathbf{4 e}$ and $\mathbf{4 g}$ exhibited activity against $E$. coli ATCC 35218 and $P$. aeruginosa at $100 \mu \mathrm{g} / \mathrm{ml}$ and against other bacteria at $50 \mu \mathrm{g} / \mathrm{ml}$. MIC values were calculated against $P$. aeruginosa as $100 \mu \mathrm{g} / \mathrm{ml}$ for compound 4d and against $E$. coli ATCC 35218 as $100 \mu \mathrm{g} / \mathrm{ml}$ for $\mathbf{4 f}$. Additionally, all compounds showed potency at the higher concentration against six bacteria E.coli ATCC 25922, E. coli ATCC 35218, E. faecalis ATCC 51299, E. faecalis ATCC 29212, $S$. aureus, K.pneumoniae. $4 \mathbf{a}$ and $\mathbf{4}$ f exhibited same potency against $P$. 
aeruginosa compared with chloramphenicol. All compounds showed lower activity against $C$. albicans than ketoconazole and they exhibited same potency against three fungal microorganisms C. krusei, C. glabrata, C. parapsilosis compared reference.

Table 1. Antimicrobial activities of the compounds ( $\mu \mathrm{g} / \mathrm{mL})$

\begin{tabular}{llllllllllll}
\hline Comp. & A & B & C & D & E & F & G & H & I & J & K \\
\hline 4a & 50 & 50 & 50 & 50 & 50 & 50 & 50 & 50 & 50 & 50 & 50 \\
4b & 50 & 100 & 50 & 50 & 50 & 50 & 100 & 50 & 50 & 50 & 50 \\
$\mathbf{4 c}$ & 50 & 100 & 50 & 50 & 50 & 50 & 100 & 50 & 50 & 50 & 50 \\
$\mathbf{4 d}$ & 50 & 50 & 50 & 50 & 50 & 50 & 100 & 50 & 50 & 50 & 50 \\
$\mathbf{4 e}$ & 50 & 100 & 50 & 50 & 50 & 50 & 100 & 50 & 50 & 50 & 50 \\
$\mathbf{4 f}$ & 50 & 100 & 50 & 50 & 50 & 50 & 50 & 50 & 50 & 50 & 50 \\
$\mathbf{4 g}$ & 50 & 100 & 50 & 50 & 50 & 50 & 100 & 50 & 50 & 50 & 50 \\
Ref. $\mathbf{1}$ & 12.5 & 12.5 & 25 & 25 & 25 & 12.5 & 50 & - & - & - & - \\
Ref. $\mathbf{2}$ & - & - & - & - & - & - & - & 25 & 50 & 50 & 50 \\
\hline
\end{tabular}

Reference 1: Chloramphenicol, Reference 2: Ketoconazole

A: E. coli ATCC 25922, B: E. coli ATCC 35218, C: E. faecalis ATCC51299, D: E. faecalis ATCC 29212, E: S. aureus, F: K. pneumonia, G: P. aeruginosa, H: $C$. albicans, I: C. krusei, J: C. glabrata, K: C. parapsilosis.

\section{REFERENCES}

1. Malladi, S.; Isloor, A.M. Synthesis and Biological Evaluation of Newer Containing Pyrazole Moiety As Antimicrobial Agents. Arab. J. Chem. 2014, 7, 1185-1191.

2. Janeczko M.; Demchuk O.M.; Strzelecka D.; Kubinski K. New Family of Antimicrobial Agents Derived From 1,4-Naphthoquinone. Eur. J. Med. Chem. 2016, 124, 1019-1025.

3. Moreira, T.; Delle, F.; Domeneghini, L.; Mascarello, A.; Regina, T.; Roberto, C.; Bardini, D.; Regina, C.; Barardi, M.; De Fatima, E.; Smânia, A.; Viancelli, A.; Ariel, L.; Garcia, T.; Augusto, R.; José, R.; Smânia, A. Antibacterial Activity of Chalcones , Hydrazones and Oxadiazoles Against Methicillin-Resistant Staphylococcus aureus. Bioorg. Med. Chem. Lett. 2012, 22, 225-230.

4. Afroz, M.; Yar M.S.; Abdel-hamid, S.G.; Al, S.I., Samad, A. Molecular Properties Prediction, Synthesis and Antimicrobial Activity of Some Newer Oxadiazole Derivatives. Eur. J. Med. Chem. 2010, 45, 12, 5862-5869.

5. Leemans, E.; Mahasenan, K.V.; Kumarasiri, M.; Spink, E.; Ding, D.; Daniel, P.I.O.; Boudreau, M.A.; Lastochkin, E.; Testero, S.A.; Yamaguchi, T.; Lee, M.; Hesek, D.; Fisher, J. F.; Chang, M.; Mobashery, S. Three-dimensional QSAR Analysis and Design of New 1, 2,4-Oxadiazole Antibacterials. Bioorg. Med. Chem. Lett. 2016, 26, 3, 1011-1015.

6. Naeem, M.; Ansar, K.; Hameed, S.; Ayub, K.; Haq, I.; Tahir, M.N.; Mahmood, T. Synthesis, Structural Studies and Biological Activities of Three New 2-(Pentadecylthio)-5-Aryl-1,3,4-oxadia- 
zoles. J. Mol. Struct. 2017, 1129, 50-59.

7. Khalilullah, H.; Khan, S.; Nomani, Md.S.; Ahmed, B. Synthesis, Characterization and Antimicrobial Activity of Benzodioxane Ring Containing 1,3,4-Oxadiazole Derivatives. Arab. J. Chem. 2011, 9, 1-7.

8. Kandemir, H.; Ma, C.; Kutty, S.K.; Black, Stc.D.; Griffith, R.; Lewis, P.J.; Kumar, N. Synthesis and Biological Evaluation of 2,5-Di(7-indolyl)-1,3,4-oxadiazoles, and 2- and 7-Indolyl 2-(1,3,4-thiadiazolyl)ketones As Antimicrobials. Bioorg. Med. Chem. 2014, 22, 5, 1672-1679.

9. Shankara, C.; Boja, P.; Sucheta, N. Synthesis, Characterization and Antimicrobial Activity of Some Disubstituted 1,3,4-Oxadiazoles Carrying 2-(Aryloxymethyl)phenyl Moiety. Eur. J. Med. Chem. 2010, 45, 4708-4719.

10. Ningaiah, S.; Bhadraiah, U. K.; Doddaramappa, S. D.; Keshavamurthy, S.; Javarasetty, C. Novel Pyrazole Integrated 1,3,4-Oxadiazoles: Synthesis, Characterization and Antimicrobial Evaluation. Bioorg. Med. Chem. Lett. 2014, 24, 1, 245-248.

11. Rane, R.A.; Bangalore, P.; Borhade, S.D.; Khandare, P.K. Oxadiazole Derivatives As Antimicrobial and Anti-tubercular Agents. Eur. J. Med. Chem. 2013, 70, 49-58.

12. Rane, R.A.; Gutte, S.D.; Sahu, N.U. Synthesis and Evaluation of Novel 1,3,4-Oxadiazole Derivatives of Marine Bromopyrrole Alkaloids As Antimicrobial Agent. Bioorg. Med. Chem. Lett. 2012, 22, 20, 6429-6432.

13. Manjunatha, K.; Poojary, B.; Lobo, P.L.; Fernandes, J.; Kumari, N.S. Synthesis and Biological Evaluation of Some 1,3,4-Oxadiazole Derivatives. Eur. J. Med. Chem. 2010, 45, 11,5225-5233.

14. Ramaprasad, G.C.; Kalluraya, B.; Kumar, B.S.; Hunnur, R.K. Synthesis and Biological Property of Some Novel 1, 3, 4-Oxadiazoles. Eur. J. Med. Chem. 2010, 45, 4587-4593.

15. Kishore, K.; Samad, A.; Kumar, Y.; Shaharyar, M.; Lal, R.; Jain, J.; Kumar, V.; Singh, P. Design, Synthesis and Biological Evaluation of 1,3,4-Oxadiazole Derivatives. Eur. J. Med. Chem. 2010, 45, 4963-4967.

16. Siddiqa, A.; Abbasi, M.A.; Rasool, S.; Siddiqui, S.Z.; Ahmad, I.; Afzal, S. Synthesis of Some New 5-Substituted-2-((6-chloro-oxadiazole Derivatives As Suitable Antibacterial Inhibitors. Bull. Fac. Pharmacy, Cairo Univ. 2015, 53, 37-43.

17. Sithambaram, M.; Jagadeesh, D.; Mahalinga, M.; Holla, B.S.; Kumari, N.S. Antimicrobial Studies of 2,4-Dichloro-5-fluorophenyl Containing Oxadiazoles. 20o8, 43, 25-31.

18. Desai, N.C.; Bhatt, N.; Somani, H.; Trivedi, A. Synthesis, Antimicrobial and Cytotoxic Activities of Some Novel Thiazole Clubbed 1,3,4-Oxadiazoles. Eur. J. Med. Chem. 2013, 67, 54-59.

19. Chandrakantha, B.; Shetty, P.; Nambiyar, V.; Isloor, N.; Isloor, A.M. Synthesis, Characterization and Biological Activity of Some New 1,3,4-Oxadiazole Bearing 2-Flouro-4-methoxy Phenyl Moiety. Eur. J. Med. Chem. 2010, 45, 1206-1210.

20. Farshori, N.N., Rauf, A., Siddiqui, M.A.; Al-Sheddi, E.S.; Al-oqail, M.M. A Facile One-Pot Synthesis of Novel Conventional and Microwave Conditions and Evaluation of Their In vitro Antimicrobial Activities. Arab. J. Chem. 2013, doi.org/10.1016/j.arabjc. 2013.11.010

21. Clinical and Laboratory Standards Institute (CLSI), Methods for Dilution Antimicrobial Susceptibility Tests for Bacteria That Grow Aerobically, Approved Standard M7-A7, CLSI,Wayne, Pa, USA, 7th edition, 2006.

(Received 21 February 2017; accepted o3 March 2017) 